\title{
Generalized description of spectral incoherent solitons
}

\author{
Gang Xu, ${ }^{1, *}$ Josselin Garnier, ${ }^{2}$ Matteo Conforti, ${ }^{3}$ and Antonio Picozzi ${ }^{1}$ \\ ${ }^{1}$ Laboratoire Interdisciplinaire Carnot de Bourgogne (ICB), CNRS-University of Burgundy, Dijon, France \\ ${ }^{2}$ Laboratoire de Probabilités et Modèles Aléatoires, University Paris Diderot, 75205 Paris Cedex 13, France \\ ${ }^{3}$ Laboratoire de Physique des Lasers, Atomes et Molécules (PhLAM), CNRS-University of Lille 1, Villeneuve d'Ascq, France \\ ${ }^{*}$ Corresponding author: gang.xu@u-bourgogne.fr
}

Received May 16, 2014; accepted June 7, 2014;

posted June 16, 2014 (Doc. ID 212158); published July 10, 2014

\begin{abstract}
Spectral incoherent solitons (SIS) refer to an incoherent solitonic structure that only can be identified in the frequency domain and which is sustained by noninstantaneous Raman-like nonlinearities. We provide a generalized formulation of SIS by considering the generalized nonlinear Schrödinger equation. We show that nonlinear dispersive effects (e.g., self-steepening) dramatically affect the structure of SIS, in particular by significantly decelerating their spectral velocity in frequency space. The wave turbulence description of the problem reveals an underlying self-organization process: the soliton trajectory in frequency space is selected in such a way that it allows the system to self-organize into a stationary SIS state. (C) 2014 Optical Society of America

OCIS codes: (190.4370) Nonlinear optics, fibers; (190.5650) Raman effect; (030.1640) Coherence.

http://dx.doi.org/10.1364/OL.39.004192
\end{abstract}

The propagation of partially coherent nonlinear optical waves is a topic of growing interest in different fields of investigations, such as, e.g., wave propagation in homogeneous [ $\underline{1}-\underline{4}]$ or periodic media []ㅡ, waveguides [6], cavity systems [ㅁ-11], supercontinuum generation (SC) [12-14], shock waves [15], or nonlinear interferometry [16]. In analogy with kinetic gas theory, an incoherent wave is expected to exhibit a nonequilibrium process of thermalization toward thermodynamic equilibrium [17]. Besides wave thermalization, a partially coherent wave can self-organize into incoherent solitons (IS), which thus constitute nonequilibrium stable states of the random field. Originally observed in photorefractive crystals [18], IS have become a blooming area of research, e.g., through the study of incoherent modulational instability $[19,20]$ or the existence of IS with spatial or temporal nonlocal nonlinearities [21-23].

An IS of a fundamentally different nature has been identified by exploiting the noninstantaneous property of the nonlinear Raman response in optical fibers [24-26]. The incoherent wave associated to this soliton exhibits fluctuations that are statistically stationary in time, so that it does not exhibit a confinement in the temporal domain but exclusively in the frequency domain; thus the name "spectral incoherent soliton" (SIS). Some experimental signatures of the generation of SIS have been obtained through the study of SC generation in photonic crystal fibers [26,27].

Our aim in this Letter is to report a nontrivial generalization of SIS structures. We consider the 1D generalized nonlinear Schrödinger equation (GNLSE), which accounts, in particular, for the self-steepening (or shock) term and a frequency dependence of the nonlinear Kerr coefficient [28-30]. Nonlinear dispersive effects are shown to dramatically affect the dynamics of the incoherent wave. Starting from the GNLSE, we derive a generalized kinetic equation (GKE), which does not admit incoherent soliton solutions. When considered as an initial condition, the standard SIS exhibits a significant deceleration in frequency space as well as a significant reduction of its power. However, the underlying soliton structure is unveiled by a change of variables, which reveals an unexpected process of self-organization. The specific form of the soliton trajectory in frequency space is selected in such a way that it allows the system to self-organize into a stationary SIS state. In this way, the spectral deceleration of SIS is obtained in analytical form and in quantitative agreement with the simulations of GNLSE, without adjustable parameters. Besides its fundamental interest, this generalized formulation of SIS can shed new light on the spectral dynamics of SC generation [26,27].

The starting point is the GNLSE, which is usually written in the frequency domain $[\underline{28}, \underline{29}]$ :

$$
\partial_{z} \tilde{\psi}_{\omega}(z)=i k(\omega) \tilde{\psi}_{\omega}-\alpha \tilde{\psi}_{\omega}(z)+i \Gamma(\omega) \mathcal{F}\left\{\psi R *|\psi|^{2}\right\},
$$

where $\mathcal{F}$ is the Fourier operator $\left[\tilde{\psi}_{\omega}(z)=\mathcal{F}\{\psi(z, t)\}=\right.$ $\left.\int \psi(z, t) \exp (i \omega t) \mathrm{d} t\right]$, while $*$ denotes the temporal convolution product between the intensity $|\psi|^{2}(z, t)$ and the response function, which includes the instantaneous (Kerr) contribution and the noninstantaneous (Raman) contribution, $R(t)=\left(1-f_{R}\right) \delta(t)+f_{R} h_{R}(t)$, where $h_{R}(t)=H(t)\left(\left(1+\eta^{2}\right) / \eta \tau_{R}\right) \sin \left(\eta t / \tau_{R}\right) \exp \left(-t / \tau_{R}\right)$, $H(t)$ being the Heaviside function $\left[\tau_{R}=32 \mathrm{fs}\right.$, $\left.\eta=\tau_{R} / \tau_{1}, f_{R} \simeq 0.18\right]$. The Taylor expansion of the dispersion relation reads $k(\omega)=\sum_{j \geq 2} \beta_{j} \omega^{j} / j$ !, with $\beta_{j}$ being the $j$-th dispersion coefficient. The function $\Gamma(\omega)=(1+p \omega) \gamma\left(\omega_{0}+\omega\right)$, with $p=1 / \omega_{0}$ ( $\omega_{0}$ being the carrier frequency), accounts for the self-steepening effect (first factor) as well as the frequency dependence of the nonlinear coefficient that arises from the frequency variation of the effective area and corresponding effective index (second factor). Note that, even if more sophisticated and accurate models have been developed for strong frequency dependence of the effective area [30], the phenomenon we are interested in is dominated by the leading self-steepening term, so we keep using the traditional and simplest form of GNLSE [Eq. (1)]. The parameter $\alpha$ accounts for the losses during propagation. If $\alpha=0$, Eq. (1) conserves the "number of photons," $N=\int|\tilde{\psi}(\omega, z)|^{2} / \Gamma(\omega) \mathrm{d} \omega$. Note that by setting $\Gamma(\omega)=\gamma_{0}$ 
where $\gamma_{0}=\gamma\left(\omega_{0}\right)$, Eq. (1) recovers the conventional nonlinear Schrödinger (NLS) equation.

It is important to recall that the typical spectral width of SIS is of the same order as the Raman gain spectral bandwidth $(\sim 13 \mathrm{THz})$. To study SIS, we thus need to avoid spectral broadening effects due to SC-like phenomena. We will thus consider the spectral dynamics of SIS far from a zero-dispersion frequency, i.e., linear dispersion effects are dominated by $\beta_{2}$. Note that, as revealed by the theory [Eq. (2)], linear dispersive effects do not play any role in the dynamics of SIS (see [31,25,26]). This shows that, in spite of their apparent similarities, the spectral deceleration of SIS discussed here is of a different nature than that discussed in the framework of a Raman-induced self-frequency shift of optical solitons $[27,29,32]$, which are inherently temporally localized coherent entities.

We report in Fig. 1 (left column) the evolution of the spectrum of the incoherent wave obtained by simulation of the GNLSE [Eq. (1)]. The initial condition is a partially coherent wave with a Gaussian spectrum and random spectral phases, superimposed on a white noise spectral background $[31,26]$. Neglecting nonlinear dispersive effects, i.e., $\overline{\Gamma(\omega)}=\gamma_{0}$, the initial incoherent wave evolves into a conventional SIS, which propagates with a constant spectral velocity $V_{0}$, and thus virtually crosses the zero-frequency component of the optical field, i.e., $\omega=-\omega_{0}$ [see Figs. 1(a)-1(d)]. Self-steepening and nonlinear dispersion regularize this "unphysical" negative frequency propagation [33] through a significant spectral deceleration of the SIS as well as a significant reduction of its power $N=\int\left|\tilde{\psi}_{\omega}\right|^{2} \mathrm{~d} \omega$ (Fig. 1). Note that
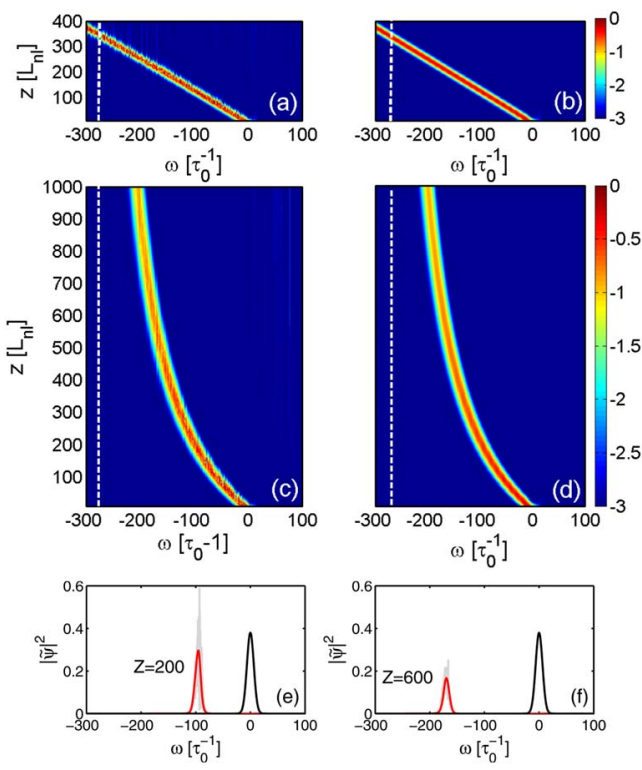

Fig. 1. Numerical simulations of the GNLSE [Eq. (1)] [left column (a) to (c)] and GKE [Eq. (2)] [right column (b) to (d)], for $p=q=0\left(\Gamma(\omega)=\gamma_{0}\right)\left[1\right.$ st row, (a) to (b)], and for $p=\omega_{0}^{-1}, q=$ $0.1 \omega_{0}^{-1}$ [2nd row, (c) to (d)]. (e) to (f) Corresponding spectral profiles obtained by simulations of GNLSE [Eq. (1)] (gray line) and GKE [Eq. (2)] (red line) at $z=200 L_{n l}$ and $z=600 L_{n l}$. The vertical dashed line denotes the physical zero frequency component, i.e., $\omega=-\omega_{0}\left[\omega_{0} /(2 \pi)=282 \mathrm{THz}\left(\lambda_{0}=1.06 \mu \mathrm{m}\right)\right.$, $\eta=2.6, \tau_{0}=\sqrt{\beta_{2} L_{n l} / 2}, L_{n l}=1 /\left(\gamma_{0} P\right)$, where $P$ is the average intensity of the incoherent wave]. the frequency reached by the SIS $\omega \sim-180 \tau_{0}^{-1}$ can correspond to a physical wavelength $\lambda \sim 3 \mu \mathrm{m}$ (for a source at $\lambda_{0} \sim 1.06 \mu \mathrm{m}$ ), where GNLSE [Eq. (1)] still can be considered as an accurate model. Also note that propagation losses have been neglected, so as to highlight the fact that the decrease of power is solely due to nonlinear dispersion effects. In this example, we considered a linear frequency variation of $\gamma$ versus $\omega, \Gamma(\omega)=$ $(1+p \omega) \gamma_{0}(1+q \omega)$, with $q=\left.\gamma_{0}^{-1} \partial_{\omega} \gamma(\omega)\right|_{\omega_{0}}$. This linear approximation also is consistent with the fact that selfsteepening is assumed to dominate the frequency dependence of the effective area in GNLSE [Eq. (1)].

To describe these novel properties of SIS, we resort to a statistical description of the random wave based on the wave turbulence theory $[1,31]$. Since we are looking for a soliton behavior, we neglect losses, $\alpha=0$ in Eq. (1). In the weakly nonlinear regime defined by $L_{d} \ll L_{n l}$ ( $L_{d} \simeq t_{c}^{2} / \beta_{2}$ being the dispersion length and $t_{c}$ the time correlation), we obtain a closure of the hierarchy of moments equations from Eq. (1), in which the averaged spectrum of the wave $\left\langle\tilde{\psi}_{\omega+\Omega / 2} \tilde{\psi}_{\omega-\Omega / 2}^{*}\right\rangle=n_{\omega}(z) \delta(\Omega)$ is governed by the following GKE:

$$
\partial_{z} n_{\omega}=\frac{n_{\omega}}{\pi} \int G\left(\omega, \omega^{\prime}\right) n_{\omega^{\prime}} \mathrm{d} \omega^{\prime}
$$

where $G\left(\omega, \omega^{\prime}\right)=\Gamma(\omega) g\left(\omega-\omega^{\prime}\right)$, and $g(\omega)=\Im[\tilde{R}(\omega)]$ is the imaginary part of the Fourier transform of $R(t)$. Note that GKE [Eq. (2)] accounts for nonlinear dispersion effects but not for linear dispersion effects (although linear dispersion is known to be essential for the establishment of a weakly nonlinear regime, which, in turn, leads to closure of the moments hierarchy [1]). Simulations of GKE [Eq. (2)] are in quantitative agreement with GNLSE without adjustable parameters, as remarkably illustrated in Fig. 1.

Because of nonlinear dispersive effects, $\Gamma(\omega) \neq \gamma_{0}$, the integral in Eq. (2) does not reduce to a convolution. For this reason, the GKE no longer conserves the power $N=\int n_{\omega} \mathrm{d} \omega$ and thus does not admit soliton solutions [34]. However, to unveil the underlying soliton dynamics, let us consider the variable change $\tilde{n}_{\omega}(z)=n_{\omega}(z) / \Gamma(\omega)$, so that GKE [Eq. (2)] can be recast into the symmetric form:

$$
\partial_{z} \tilde{n}_{\omega}=\frac{1}{\pi} \int \tilde{n}_{\omega} \Gamma(\omega) g\left(\omega-\omega^{\prime}\right) \Gamma\left(\omega^{\prime}\right) \tilde{n}_{\omega^{\prime}} \mathrm{d} \omega^{\prime}
$$

This modified GKE (MGKE) conserves the power $\tilde{N}=\int \tilde{n}_{\omega} \mathrm{d} \omega$ and can thus exhibit soliton solutions. However, as revealed by the simulations in Fig. 1 , such soliton solutions are not conventional, in the sense that they do not propagate with a constant velocity in frequency space. We thus look for a stationary soliton solution in a "noninertial" reference frame in frequency space, i.e., a reference frame travelling with a nonstationary (z-dependent) spectral velocity $V(z)=\partial_{z} \Omega^{s}(z): \Omega=$ $\omega-\Omega^{s}(z)$. The analysis will reveal that the soliton trajectory, $\Omega^{s}(z)$, is selected in such a way that it allows the system to self-organize into a stationary SIS state. In the noninertial reference frame, the MGKE [Eq. (3)] reads: 


$$
\begin{aligned}
\partial_{z} \tilde{n}_{\Omega}-\partial_{z} \Omega^{s}(z) \partial_{\Omega} \tilde{n}_{\Omega}= & \frac{\tilde{n}_{\Omega}}{\pi} \int g\left(\Omega-\Omega^{\prime}\right) \tilde{n}_{\Omega^{\prime}} \\
& \times \Gamma\left[\Omega+\Omega^{s}(z)\right] \Gamma\left[\Omega^{\prime}+\Omega^{s}(z)\right] \mathrm{d} \Omega^{\prime},
\end{aligned}
$$

where $\Omega^{\prime}=\omega^{\prime}-\Omega^{s}(z)$. In general, one cannot decouple Eq. (4) into two independent equations for $\tilde{n}_{\Omega}(z)$, and for the soliton trajectory $\Omega^{s}(z)$. However, the SIS profile and $g(\omega)$ vary on a frequency scale much smaller than $\Gamma(\omega)$. We thus introduce the multiscale expansion $\tilde{n}_{\Omega}(z)=\varepsilon^{-1} m_{\Omega / \varepsilon}(z)$ and $g(\omega)=\varepsilon^{-1} \tilde{g}(\omega / \varepsilon)$ and look for a solution of Eq. ( $\underline{4})$ in the form, $m_{\bar{\Omega}}(z)=m_{\bar{\Omega}}^{s}+$ $\varepsilon \delta m_{\bar{\Omega}}(z)$, where $m_{\bar{\Omega}}^{s}$ is the stationary soliton profile in the noninertial reference frame and $\delta m_{\bar{\Omega}}(z)$ is a residual radiation $(\bar{\Omega}=\Omega / \varepsilon)$. To the first order in $\varepsilon$, Eq. (4) then decouples into two independent equations for the soliton profile, $\tilde{n}_{\Omega}^{s}=\varepsilon^{-1} m_{\Omega / \varepsilon}^{s}$, and the soliton trajectory:

$$
\begin{aligned}
& \partial_{z} \Omega^{s}(z)=-V_{0} \Gamma^{2}\left(\Omega^{s}(z)\right) / \gamma_{0}^{2}, \\
& V_{0} \partial_{\Omega} \tilde{n}_{\Omega}^{s}=\frac{\gamma_{0}^{2}}{\pi} \tilde{n}_{\Omega}^{s} \int g\left(\Omega-\Omega^{\prime}\right) \tilde{n}_{\Omega^{\prime}}^{s} \mathrm{~d} \Omega^{\prime} .
\end{aligned}
$$

Here, by introducing $V_{0}(>0)$, we have recast Eq. (6) into the usual stationary equation for a conventional SIS with $\Gamma(\omega)=\gamma_{0}$, which propagates with the constant velocity $V_{0}$. This reveals a remarkable result: within the new variable $\tilde{n}_{\omega}=n_{\omega} / \Gamma(\omega)$, the SIS spectral profile is almost identical to the conventional SIS spectral profile for $\Gamma(\omega)=\gamma_{0}$, while the corresponding soliton trajectory, $\Omega^{s}(\boldsymbol{z})$, satisfies the differential in Eq. (5). Note that the multiscale expansion also readily gives the power decay:

$$
N(z)=\tilde{N} \Gamma\left[\Omega^{s}(z)\right],
$$

where $\tilde{N}=\int \tilde{n}_{\omega}^{s} \mathrm{~d} \omega$ is the conserved power of MGKE [Eq. (3)] and $\Omega^{s}(z)$ is obtained in explicit form in Eq. (8). The power decay in Eq. (7) is found in quantitative agreement with simulations, as illustrated in Fig. 2(b).

The soliton trajectory can be obtained analytically under the assumption of a linear frequency dependence, $\gamma\left(\omega_{0}+\omega\right)=\gamma_{0}(1+q \omega)$. In this case, Eq. (5) takes the form $\int_{0}^{-\Omega^{s}}(1-q u)^{-2}(1-p u)^{-2} \mathrm{~d} u=V_{0} z$, with $p=1 / \omega_{0}$ and $\Omega^{s}(z=0)=0$. The solution $z$ versus $\Omega^{s}$ reads:

$$
z\left(\Omega^{s}\right)=\frac{1}{V_{0}(q-p)^{3}}\left[2 p q \log \left(\frac{1+q \Omega^{s}}{1+p \Omega^{s}}\right)-Q_{q p}\left(\Omega^{s}\right)\right],
$$

where $Q_{q p}\left(\Omega^{s}\right)=(q-p)\left[q p(q+p) \Omega^{s}+q^{2}+p^{2}\right] \Omega^{s} /$ $\left[\left(1+q \Omega^{s}\right)\left(1+p \Omega^{s}\right)\right]$. Note that, by neglecting nonlinear dispersive effects, i.e., $\Gamma(\omega)=\gamma_{0}$ (i.e., $p=q=0$ ), Eq. (8) reduces to the linear trajectory, $\Omega^{s}(z)=-V_{0} z$, inherent to conventional SIS $[31,24,26]$. Conversely, whenever $\Gamma(\omega) \neq \gamma_{0}, \Omega^{s}(z)$ asymptotically reaches $-\omega_{0}$ for large propagation lengths $z$ (see Fig. 2), which regularizes the unphysical negative frequency propagation predicted by the linear trajectory, $\Omega^{s}(z)=-V_{0} z$.

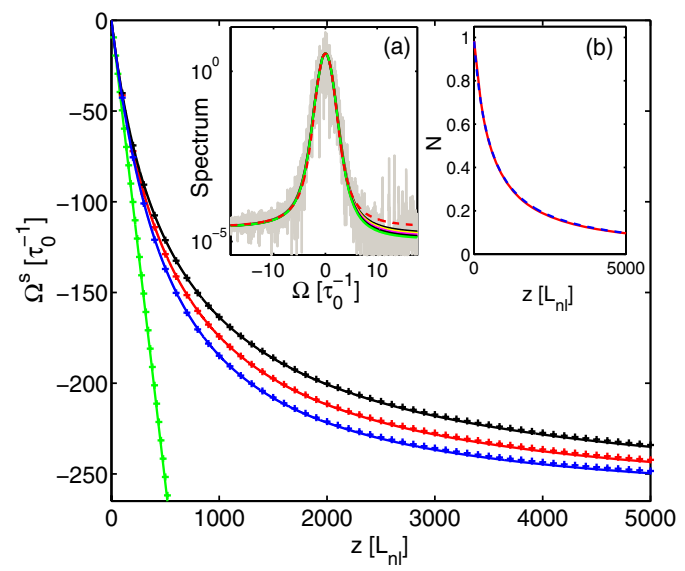

Fig. 2. Soliton trajectory $\Omega^{s}$ versus $z$ plotted from Eq. (8), in which $V_{0}$ is given by Eq. (10): $q=p=0, \Omega^{s}(z)=-V_{0} z$ (green); $q=0, p=\omega_{0}^{-1}$ (red); $q=0.2 p, p=\omega_{0}^{-1}$ (black); $q=-0.2 p, p=$ $\omega_{0}^{-1}$ (blue). The corresponding crosses on the curves refer to the simulations of MGKE [Eq. (3)]; a quantitative agreement is obtained without adjustable parameters. The inset (a) compares the simulations of the MGKE [Eq. (3)] (green) and GNLSE [Eq. (1)] (gray) with the analytical SIS solution in Eqs. (9) and (10), in $\log _{10}$-scale. The superposition of five spectral profiles at $z=200,400,600,800,1000$ (in units of $L_{n l}$ ) has been plotted to stress the stationary character of the SIS solution obtained by simulation of MGKE [Eq. (3)]. The inset (b) shows the power decrease, $N=\int\left|\psi_{\omega}\right|^{2} \mathrm{~d} \omega$ versus $z$, in the GNLSE [Eq. (1)] (red), and the theoretical prediction in Eq. (7) (blue dashes) $\left[\omega_{0} /(2 \pi)=282 \mathrm{THz}, \eta=1\right]$.

An analytical expression of SIS solution with velocity $V_{0}$ was obtained in [24] through a generalization of the particular solution originally derived in [35] in the limit $n_{0} / n_{m} \ll 1$, where $n_{m}$ and $n_{0}$, respectively, denote the soliton peak and the level of the background noise. Note that so far this SIS solution has not been compared with numerical simulations. The solution reads:

$$
\tilde{n}_{\Omega}^{s}=n_{0} \exp \left[\log \left(\frac{n_{m}}{n_{0}}\right) \frac{H(\Omega)}{g_{\mathrm{i}} \omega_{\mathrm{i}}}\right],
$$

where $g_{\mathrm{i}}=\left[\left|\partial_{\omega} g(0)\right| H(0)\right]^{1 / 2}$ denotes the typical amount of gain of $g(\omega), \omega_{\mathrm{i}}=\left[\left|\partial_{\omega} g(0)\right| H(0)\right]^{1 / 2}$ its typical spectral bandwidth, and $H(\Omega)=-\int_{\Omega}^{\infty} g(\omega) \mathrm{d} \omega$. The SIS propagates with the constant velocity:

$$
V_{0}=\frac{\gamma_{0}^{2}}{\pi} \frac{\int\left(\tilde{n}_{\Omega}^{s}-n_{0}\right) \mathrm{d} \Omega}{\int \log \left(\tilde{n}_{\Omega}^{s} / n_{0}\right) \mathrm{d} \Omega} \int H(\Omega) \mathrm{d} \Omega .
$$

We have compared the expression of the soliton trajectory in Eq. (8) as well as the corresponding SIS solution in Eqs. (9) and (10) with the results of the numerical simulations of the MGKE [Eq. (3)] and NLS [Eq. (1)] in the weakly nonlinear regime $\left(L_{d} \ll L_{n l}\right)$. A quantitative agreement has been obtained without adjustable parameters, as remarkably illustrated in Fig. 2. In the limit of a small SIS spectral shift, the trajectory recovers the straight line, $\Omega^{s}(z)=-V_{0} z$ of conventional SIS, where $V_{0}$ is given by Eq. (10). In the limit $q=0$ $(\Gamma(\omega)=1+p \omega)$, the SIS trajectory takes the simple form $\Omega^{s}(z)=-V_{0} z /\left(1+V_{0} z / \omega_{0}\right)$ (red line in Fig. 2). A positive (negative) value of $q$ then decelerates (accelerates) 

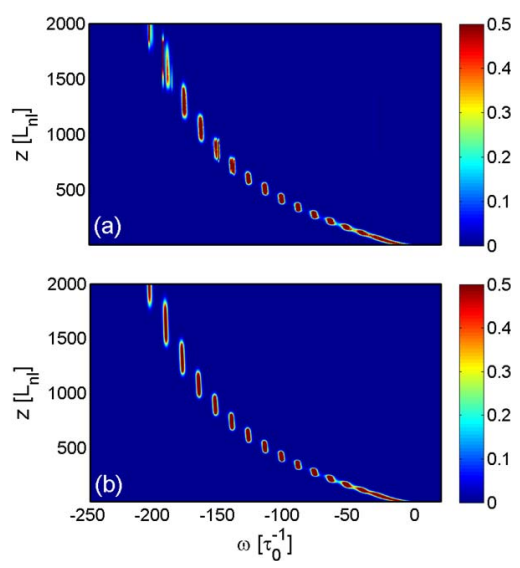

Fig. 3. Transition to a discrete behavior of generalized SIS in the presence of a small noise background, $n_{0} / n_{m}^{0}=10^{-7}, n_{m}^{0}$ being the initial spectral peak amplitude. Simulations of the (a) GNLSE [Eq. (ㅁ)] and (b) GKE [Eq. (2)] $\left[\omega_{0} /(2 \pi)=\right.$ $282 \mathrm{THz}, \eta=2.6, p=\omega_{0}^{-1}, q=0.1 \omega_{0}^{-1}$ ].

the spectral shift of the SIS (see black and blue lines in Fig. 2). Finally, the plot of the analytical SIS solution in Eqs. (9) and (10) has been found in quantitative agreement with simulations of MGKE [Eq. (3)] and GNLSE [Eq. (1)], as illustrated in Fig. 2(a).

We have provided a generalized wave turbulence formulation of incoherent optical waves and SIS, accounting for nonlinear dispersive effects. Note that, as the spectral noise background decreases, the generalized SIS exhibits a transition to a discrete behavior (see Fig. 3) in a way similar to conventional SIS [25], a feature that will be the subject of future investigations.

This work has been supported by the Labex ACTION program (contract ANR-11-LABX-01-01) and the French National Research Agency (ANR-12-BS04-0011 OPTIROC).

\section{References}

1. A. Picozzi, J. Garnier, T. Hansson, P. Suret, S. Randoux, G. Millot, and D. Christodoulides, "Optical wave turbulence: toward a unified nonequilibrium thermodynamic formulation of statistical nonlinear optics," Phys. Rep., http://dx .doi.org/10.1016/j.physrep.2014.03.002 (to be published).

2. T. Hansson, D. Anderson, M. Lisak, V. E. Semenov, and U. Österberg, J. Opt. Soc. Am. B 25, 1780 (2008).

3. A. Piskarskas, V. Pyragaite, and A. Stabinis, Phys. Rev. A 82, 053817 (2010).

4. J. Laurie, U. Bortolozzo, S. Nazarenko, and S. Residori, Phys. Rep. 514, 121 (2012).

5. Y. Silberberg, Y. Lahini, Y. Bromberg, E. Small, and R. Morandotti, Phys. Rev. Lett. 102, 233904 (2009).

6. P. Aschieri, J. Garnier, C. Michel, V. Doya, and A. Picozzi, Phys. Rev. A 83, 033838 (2011).
7. C. Conti, M. Leonetti, A. Fratalocchi, L. Angelani, and G. Ruocco, Phys. Rev. Lett. 101, 143901 (2008).

8. S. Babin, D. Churkin, A. Ismagulov, S. Kablukov, and E. Podivilov, J. Opt. Soc. Am. B 24, 1729 (2007).

9. E. Turitsyna, S. Smirnov, S. Sugavanam, N. Tarasov, X. Shu, S. Babin, E. Podivilov, D. Churkin, G. Falkovich, and S. Turitsyn, Nat. Photonics 7, 783 (2013).

10. R. Weill, B. Fischer, and O. Gat, Phys. Rev. Lett. 104, 173901 (2010).

11. A. Schwache and F. Mitschke, Phys. Rev. E 55, 7720 (1997).

12. B. Barviau, B. Kibler, and A. Picozzi, Phys. Rev. A 79, 063840 (2009).

13. B. Barviau, J. Garnier, G. Xu, B. Kibler, G. Millot, and A. Picozzi, Phys. Rev. A 87, 035803 (2013).

14. M. Erkintalo, M. Surakka, J. Turunen, A. T. Friberg, and G. Genty, Opt. Lett. 37, 169 (2012).

15. J. Garnier, G. Xu, S. Trillo, and A. Picozzi, Phys. Rev. Lett. 111, 113902 (2013).

16. S. Derevyanko and E. Small, Phys. Rev. A 85, 053816 (2012).

17. P. Suret, S. Randoux, H. R. Jauslin, and A. Picozzi, Phys. Rev. Lett. 104, 054101 (2010).

18. M. Mitchell, Z. Chen, M. Shih, and M. Segev, Phys. Rev. Lett. 77, 490 (1996).

19. M. Soljacic, M. Segev, T. Coskun, D. N. Christodoulides, and A. Vishwanath, Phys. Rev. Lett. 84, 467 (2000).

20. B. Kibler, C. Michel, J. Garnier, and A. Picozzi, Opt. Lett. 37, 2472 (2012).

21. C. Rotschild, T. Schwartz, O. Cohen, and M. Segev, Nat. Photonics 2, 371 (2008).

22. A. Picozzi and J. Garnier, Phys. Rev. Lett. 107, 233901 (2011).

23. C. Michel, B. Kibler, J. Garnier, and A. Picozzi, Phys. Rev. A 86, 041801(R) (2012).

24. J. Garnier and A. Picozzi, Phys. Rev. A 81, 033831 (2010).

25. C. Michel, B. Kibler, and A. Picozzi, Phys. Rev. A 83, 023806 (2011).

26. B. Kibler, C. Michel, A. Kudlinski, B. Barviau, G. Millot, and A. Picozzi, Phys. Rev. E 84, 066605 (2011).

27. G. P. Agrawal, Nonlinear Fiber Optics, 5th ed. (Academic, 2012).

28. K. J. Blow and D. Wood, IEEE J. Quantum Electron. 25, 2665 (1989).

29. J. Travers, M. Frosz, and J. Dudley, "Nonlinear fibre optics overview," in Supercontinuum Generation in Optical Fibers, J. Dudley and J. R. Taylor, eds. (Cambridge University, 2010), p. 32.

30. J. Laegsgaard, Opt. Express 15, 16110 (2007).

31. A. Picozzi, S. Pitois, and G. Millot, Phys. Rev. Lett. 101, 093901 (2008).

32. A. A. Voronin, A. M. Zheltikov, and M. Aleksei, Opt. Lett. 33, 1723 (2008).

33. M. Conforti, A. Marini, T. X. Tran, D. Faccio, and F. Biancalana, Opt. Express 21, 31239 (2013).

34. S. L. Musher, A. M. Rubenchik, and V. E. Zakharov, Phys. Rep. 252, 177 (1995).

35. C. Montes, Phys. Rev. A 20, 1081 (1979). 